Check for updates

Cite this: RSC Adv., 2019, 9, 6596

\title{
Electro-deposition for asphaltene removal during heavy oil upgrading
}

\author{
Shunxiang Xia, Enjelia Veony and Konstantinos Kostarelos (DD*
}

Blending crude oil with short-chain paraffins is a common method to improve the oil quality during heavy oil upgrading. The additional paraffins will cause precipitation of asphaltene that is removed by filtration or sedimentation; both processes are slow and inefficient. As a potential faster and more efficient removal method, an electric field can be applied in order to electro-deposit the asphaltene on the electrodes. Electro-deposition (E-D) experiments were conducted in a bench scale vessel while varying several process parameters such as the dilution ratio of paraffin to heavy oil, the paraffin used as the diluent, electric field strength, and the effect of resins on the E-D process. Increasing the dilution ratio resulted in more precipitated asphaltene and required a lower electric field strength for the E-D process. The electro-deposition process could affect the net charge of the asphaltene, and hence, both cathode and anode were closely observed: deposition onto the cathode was favored with higher dilution ratio and a lower electric field strength, while anode deposition occurred using a lower dilution ratio and higher electric field strength. This result is related to the higher resin content at low dilution ratio which adsorbs onto the asphaltene aggregate and shields or inhibits the effect of the electric field. To recover $1 \mathrm{~kg}$ of asphaltene, the energy input is estimated to be as low as $10 \mathrm{~kJ}$. The process is relatively fast and requires low energy input, which can overcome the disadvantages of current filtration methods.

Received 23rd December 2018 Accepted 19th February 2019

DOI: 10.1039/c8ra10514f

rsc.li/rsc-advances blockages. ${ }^{9}$ However, both filtration and sedimentation are timeconsuming processes and, moreover, inefficient to deal with low asphaltenes content oil and when the asphaltene aggregates are too small to be retained by the filtration membrane. ${ }^{10}$

Although it has been known that asphaltenes behave as though net charged molecules, ${ }^{8-10}$ the use of electro-deposition for asphaltenes removal is rarely mentioned in the literature. The net charge of asphaltenes is believed to come from the $\pi$ bonds in the chemical structure and hetero-atoms such as $\mathrm{Ni}$, $\mathrm{Vi}, \mathrm{N}, \mathrm{O}$ and $\mathrm{S},{ }^{11,12}$ while a detailed structure for them is still unclear. Only a very recent paper utilizing atomic force microscopy (AFM) provides images of the molecules ${ }^{13,14}$ and most of our understanding has come from indirect observations. ${ }^{15}$ Under an electric field, an electrostatic force is applied to the precipitated asphaltene that is in direct proportion to the charge of the particle and the electric field strength and results in motion. At the same time, the friction (viscous) drag force increases with the acceleration of the particle until it equals the electrostatic force and the particle reaches terminal velocity (eqn (1)).

$$
v=E q / 6 \pi \mu r
$$

where $v$ is the velocity of the particle; $E$ is the electric field strength; $q$ is the amount of the charge; $\mu$ is the viscosity of fluid; $r$ is the radius of particle.

For this proposed application where an E-D approach would be used to remove the precipitated asphaltenes from a flow 
stream, the electric field strength needed to be strong enough to allow all the asphaltenes to deposit while traveling passed the electrode (i.e., residence time within the E-D device). Different from filtration methods, E-D is more efficient to deal with small asphaltene aggregates when considering that the electrophoretic mobility of the asphaltene aggregates is higher. To develop this concept, the parameters that may affect the electrophoretic mobility of asphaltenes need to be well-understood. The electrophoresis of asphaltenes can be affected by both the characteristics of the asphaltenes (e.g., elemental composition, surface charge, aggregate size) and the process parameters such as electric field strength, diluent type, dilution ratio, and residence time. ${ }^{16-18}$

The precipitation of asphaltenes due to the addition of paraffin is a critical initial step for the success of the proposed E-D application. According to the literature, only precipitated asphaltenes exhibit electric-kinetic behavior. ${ }^{16,19}$ As the charge of asphaltenes is believed to come from the $\pi$-bonding and metallo-porphyrin groups such as nickel porphyrin and vanadium porphyrin, the assembled structure of asphaltenes aggregates may be necessary to maintain the charge and result in electro-deposition. When varying the ratio of alkane (heptane) to aromatic (toluene), Khvostichenko (2009) observed an apparent change in the polarity of the asphaltenes: single anode deposition was observed with a heptane fraction below $40 \%$, while both anode and cathode deposition occurred at higher heptane fraction. ${ }^{\mathbf{1 6}}$ It is well-known that the asphaltenes in crude oil represent a group of molecules and not a single, pure chemical species; the variety of asphaltenes possess different molecular weight, elemental composition, and more importantly, degree of polarity. ${ }^{20}$ Thus, a wide range in electrophoretic mobility was observed using asphaltenes from the same source and an above-average electric field strength was suggested by Hashmi to guarantee the complete electrodeposition of asphaltenes. ${ }^{\mathbf{1 1}}$ Studies have also shown that the presence of resin can reduce its electrophoretic mobility, presumably by partially neutralizing the net charge of asphaltenes. ${ }^{21,22}$ Different from asphaltenes, resin is defined as heavy component of crude oil and can dissolve in both paraffins and aromatics. Moreover, resin is believed to possess the opposite surface charge of asphaltene, which explains their interaction with asphaltene, ${ }^{23}$ although the mechanism of interaction between resin and asphaltenes is still debatable. Nellensteyn believed that resin coated the surface of asphaltene aggregates, ${ }^{24}$ while later research indicated that resin formed coaggregates with asphaltenes. ${ }^{25}$ Regardless of the mechanism, the point is made that the existence of resin can significantly affect the E-D performance.

With the aim of developing an E-D based strategy for asphaltene removal during oil upgrading, the electric field strength requirements were measured for two crude oil blends in order to compare the effect of asphaltene and resin content. Various process parameters such as diluent types, diluent: oil ratio (oil volume fraction), and the existence of resin were studied, and all affected the electrophoretic mobility of the asphaltenes, and more importantly, the apparent charge of the asphaltene.
To complete the process, the total energy input is the sum of energy input for building up the electric field strength $\left(E_{\mathrm{b}}\right.$, eqn (2)) and energy consumption during the electrodeposition $\left(E_{\mathrm{m}}\right.$, eqn (3)).

$$
E_{\mathrm{b}}=\frac{1}{2} C V^{2} \text { and } C \approx \varepsilon A / d \text { when } A \gg d^{2}
$$

where $C$ is the capacitance; $V$ is the electric field strength; $\varepsilon$ is the permittivity of a dielectric; $A$ is the area of electrode; $d$ is the distance between the electrode.

$$
E_{\mathrm{m}}=V I t
$$

where $I$ is the average current during the process and $t$ is the process time. With low current and capacitance, the energy input is extremely low. The high energy efficiency and simple operation make the E-D approach a promising method to remove asphaltenes during the oil upgrading process.

\section{Material and methods}

\section{Material}

Two blended crude oil samples were used in this work: one blend is characterized by a high asphaltene and resin content (HAS) while the second blend is considered as having low asphaltene and resin content (LAS). Both blends were prepared by mixing a heavy oil extracted from Canadian oil sands (Alberta Innovates, Canada) and Permian basin light oil at two blend ratios by mass: $1: 4$ for HAS and $1: 20$ for LAS. The blended oil samples were prepared for testing by diluting with one of three paraffins: pentane, hexane, and heptane, all purchased from Alfa Aesar (Haverhill, USA).

The standard chromatographic method ASTM D2007-93 was used for the SARA (saturates, aromatics, resin, and asphaltene) analysis of both HAS and LAS and the results are shown in Table 1. The viscosity of oil sample was measured with CannonFenske viscometer (Kimax, USA) at room temperature $\left(25^{\circ} \mathrm{C}\right)$.

\section{Mass of asphaltenes and associated resin}

The crude oil blend was diluted at various diluent : oil ratios (volumetric) using either pentane, hexane, or heptane and shaken for $5 \mathrm{~min}$ by hand, after which the precipitates were allowed to settle for 6 hours at room temperature. The precipitates from each $10 \mathrm{~mL}$ oil/paraffin solution were collected after centrifuging at $8000 \mathrm{rpm}$ for $10 \mathrm{~min}$; then, the precipitates were dispersed and washed with $10 \mathrm{~mL}$ of pentane 3 times (total 30 $\mathrm{mL}$ ) to dissolve and remove any associated resins. The washed asphaltenes were dried at $50{ }^{\circ} \mathrm{C}$ overnight and then dissolved in $10 \mathrm{~mL}$ of toluene. The concentration was measured with nearinfrared (NIR) spectroscopy (NIR Quest 512-2.5, Ocean Optics, Inc., USA) by monitoring the absorption value at $1700 \mathrm{~nm}$ wavelength (Fig. 1) to calculate the mass of precipitated asphaltene.

The concentration of resin dissolved in the $30 \mathrm{~mL}$ pentane "wash" was also determined by spectrophotometer at $430 \mathrm{~nm}$ (Vernier Colorimeter COL-BTA, Vernier, Inc., USA) to calculate the amount of resin associated with the precipitated 
Table 1 Properties of HAS and LAS crude oils used in this work

\begin{tabular}{lllllll}
\hline & Saturates wt $\%$ & Aromatics wt $\%$ & Resin wt $\%$ & Asphaltenes wt\% & ${ }^{\circ}$ API gravity & Viscosity cP \\
\hline HAS & 71.2 & 14.9 & 4.5 & 9.3 & 37.6 & 27.3 \\
LAS & 81.5 & 12.8 & 1.5 & 4.2 & 44.8 & 16.1
\end{tabular}

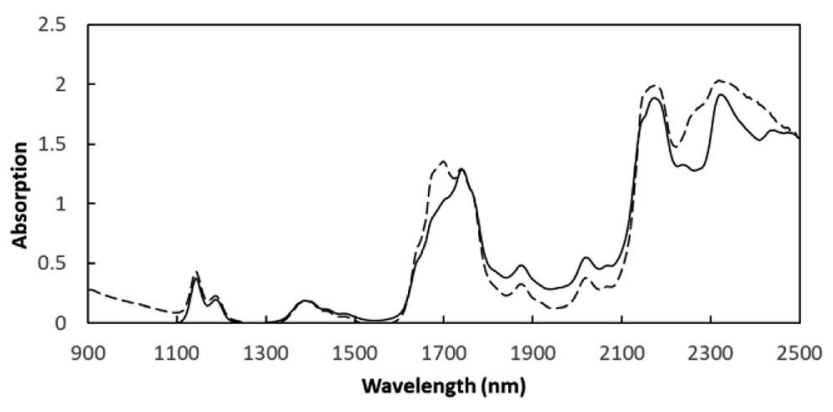

Fig. 1 The NIR spectrum of asphaltenes in toluene (dashed line: $1 \mathrm{~g} \mathrm{~L}^{-1}$ asphaltene dissolved in toluene; solid line: pure toluene without asphaltene).

asphaltenes. Knowing the mass of precipitated asphaltene and associated resin, the mass ratio of resin to asphaltene can be calculated for various dilution ratios.

\section{Electro-deposition experiments}

The E-D experiments were conducted in a cell fabricated at the University of Houston using a Teflon ${ }^{\circledR}$ cylinder fitted with two removable, parallel copper disks that functioned as electrodes (a photograph of the electrodes appears in Fig. 3). The electrodes have a diameter of $4 \mathrm{~cm}$ and the separation distance between two electrodes was fixed at $1.5 \mathrm{~cm}$. A DC electric field was generated with a high voltage power supply (PS375 Stanford Research Systems, Inc., USA); the electric field strength in all experiments can be adjusted between $0-12000 \mathrm{~V} \mathrm{~cm}^{-1}$. During the E-D process, the current across the electrodes was recorded using a multimeter (Fluke 289, Fluke Corporation, USA).

Upon adding diluent, $10 \mathrm{~mL}$ of the diluted crude oil was quickly moved into the cell for the experiment and the electric field applied for a duration of exactly one minute. After that, the residual liquid was carefully removed from the cell and the mass of un-deposited asphaltenes was determined with the same NIR method described above to check whether the electric deposition was "complete" (recover more than 90\% precipitated asphaltenes).

The image of the electro-deposited material was captured by a Canon SX510 digital camera (Canon, Japan); the SEM image was captured by JSM 6400 Scanning Electron Microscope (JEOL, Japan).

\section{Results and discussion}

\section{Asphaltenes precipitated with various diluent fraction}

To meet pipeline assurance requirements, diluent to crude oil ratios can vary from $10: 1$ to $1: 10 .^{26}$ In this study, the high range in terms of diluent fraction was the focus to evaluate the asphaltenes removal efficiency. While the SARA analysis reports the total resin and asphaltene content of each crude oil, only the asphaltenes that have precipitated can be affected by the E-D process; thus, it is important to quantify the amount of asphaltenes that can be precipitated from the crude oil samples upon paraffin addition in order to understand the efficiency of the E-D process. It was also of interest to understand which of three paraffins resulted in greatest asphaltene precipitation. The mass fractions of asphaltenes precipitated with various oil volume fraction for both HAS and LAS were determined and are shown in Fig. 2.

It is interesting to note in Fig. 2 that for the LAS, 87.5, 77.1 and $72.2 \mathrm{wt} \%$ of asphaltenes were precipitated with $95 \mathrm{vol} \%$ pentane, hexane and heptane, respectively, and that these values decreased to $62.5,54.7$ and 44.9 wt $\%$ with 75 vol\% pentane. In this study, about $10 \mathrm{wt} \%$ of asphaltenes remained dissolved in the fluid with 90 vol\% pentane, and further increasing the dilution ratio resulted in trivial improvement of precipitation. Since it is known that the more polar asphaltenes require less dilution in order to precipitate, it is likely that the last $10 \mathrm{wt} \%$ of dissolved asphaltenes were the least polar. We
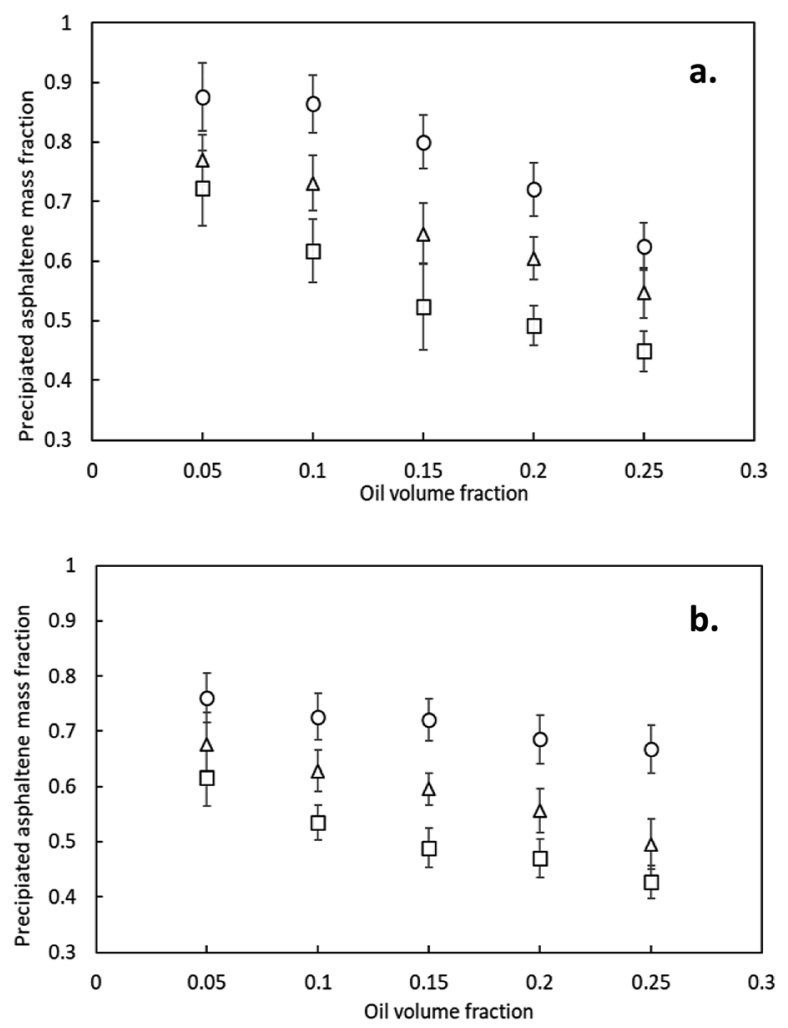

Fig. 2 The mass of asphaltenes precipitated upon dilution of crude oil with paraffin as a fraction of total asphaltenes: pentane (circle), hexane (triangle), and heptane (square). (a) LAS crude oil blend; (b) HAS crude oil blend. 
should point out that, although this fraction of asphaltenes would be difficult to remove by means of precipitation and subsequent electro-deposition, this fraction also tends to remain dissolved and stable during the upgrading process and will not likely lead to fouling of piping and equipment.

The three paraffins used to cause asphaltenes precipitation possess very low degree of polarity; the relative polarity of pentane, hexane, and heptane were 0.009, 0.009, and 0.012 (D/ D), respectively. ${ }^{27}$ The addition of pentane to crude oil exhibited a better effect than the other two, which is logical since it is the least polar. To achieve $70 \mathrm{wt} \%$ asphaltenes removal, the volume fraction of pentane, hexane and heptane were $78 \%, 88 \%$ and $93 \mathrm{vol} \%$ respectively, illustrating the sensitivity of asphaltenes precipitation to the polarity of the diluent. It should also be noted that a higher diluent fraction was required for asphaltene deposition of the HAS than LAS; the higher content of resin and aromatics (see Table 1) in the HAS function to balance polarity of the asphaltenes against precipitation during the dilution process. ${ }^{28,29}$ Based on these observations, pentane was chosen as the diluent to be used in this study for the further research.

\section{Resin associated with precipitated asphaltenes}

During the dilution process, asphaltenes with accompanied resin are precipitated out of liquid phase. The amount of resin associated with precipitated asphaltenes for various diluent : oil ratio (pentane as diluent) was measured and the data shown in Fig. 3.

As shown in Fig. 3, the mass ratio between associated resin and precipitated asphaltenes was between 0.13 and 0.69 ; also note that the mass ratio increases with higher oil volume fraction for both crude oil blends. As an example, for LAS, for each gram of precipitated asphaltenes, $0.13 \mathrm{~g}$ and $0.47 \mathrm{~g}$ of resin was associated with $5 \mathrm{vol} \%$ and $25 \mathrm{vol} \%$ of oil fractions, respectively. As oil fraction is increased, the precipitated asphaltenes is more polar, which can have more associated resin. Compared with LAS, the asphaltenes of the HAS blend tended to associate with more resin: with $25 \mathrm{vol} \%$ of oil, $0.69 \mathrm{~g}$ of associated resin per gram of precipitated asphaltenes, which is $50 \%$ more than that in LAS. It should be noted that this comparison differs only in the ratio used to blend the two crude oils. The effect of resin association on the performance of E-D can be summarized as

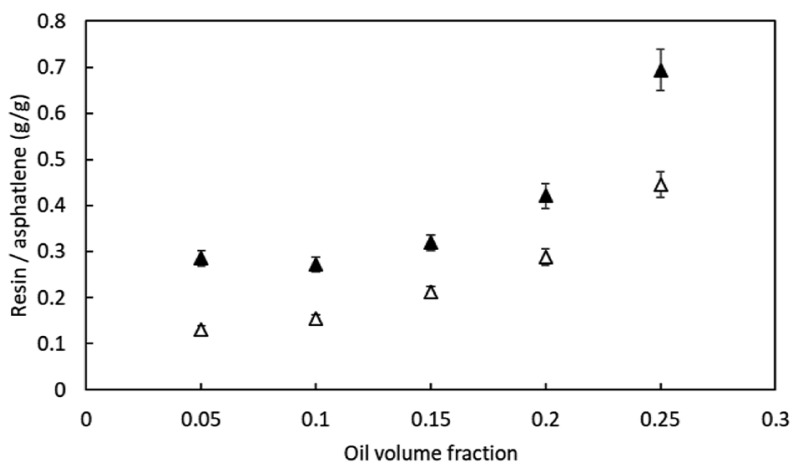

Fig. 3 The amount of resin associated with precipitated asphaltenes for various diluent : oil ratio (as oil fraction); white: LAS crude oil; black: HAS crude oil. following: the bound resin increases the mass of aggregated asphaltenes and decreases the electrophoretic mobility of aggregates under the electric field, and moreover; the polarity of resin may neutralize and perhaps alter the net charge of asphaltene aggregate, thereby affecting the electrode upon which the deposition will occur.

\section{The net charge of asphaltenes during electro-deposition}

Depending upon the net charge of the asphaltene aggregates (associated with resin), deposition upon either the cathode, anode or both may occur during the E-D process. Examples of cathode and anode deposition were photographed and shown in Fig. 4.

In Fig. 4, the asphaltenes (contain resin) formed a firm deposition on the surface of electrode and no textural difference was found between material deposited on the anode and cathode. Note that the test vessel is approximately half-filled so that electro-deposition can only occur on the lower half of the electrodes. Different from Langmuir adsorption without an electric field, multiple layers were observed in our study and the resin appeared to function as an adhesive material and adhere several layers (Fig. 4(c)). According to Azari's study, the electrode can be refreshed through detachment of deposited asphaltene by reversing the electric field; ${ }^{30}$ at this point, the asphaltenes can be collected and recycled for a beneficial use such as chemicals synthesis or energy generation. ${ }^{31,32}$

The net charge of the deposited asphaltene aggregates was evidenced by the electrode upon which they deposited and the observations are summarized in Table 2 . The parameters being varied include electric field strength, oil fraction, and the asphaltenes and resin content; all affect the net charge of the aggregate and hence the electrode upon which deposition was observed. First, note that increasing the electric field strength can trigger the anode deposition. With oil fraction fixed at $15 \mathrm{vol} \%$, only cathode deposition was observed when the electric field strength was low, but elevating the electric field strength to 9333 $\mathrm{V} \mathrm{cm}{ }^{-1}$, resulted in deposition onto both electrodes. Next, note that cathode deposition is preferred at high diluent: oil ratio (low oil fraction); as the oil fraction was increased from 10 to

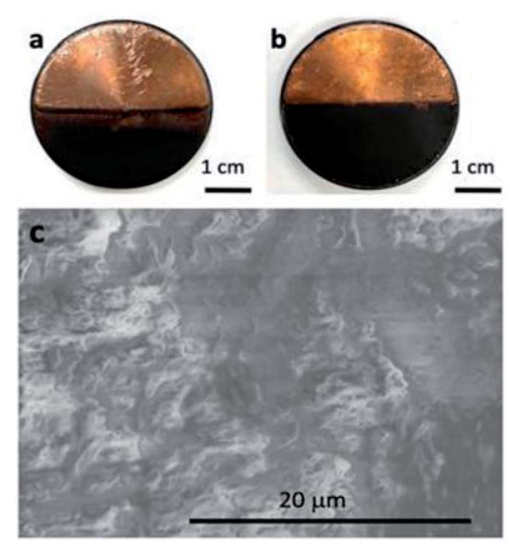

Fig. 4 Photograph of both (a) anode and (b) cathode deposition and (c) the SEM image of cathode deposition for 15 vol\% LAS diluted with pentane under $9333 \mathrm{~V} \mathrm{~cm}^{-1}$. 
Table 2 The effect of operating parameters (field strength, oil fraction, and asphaltene and resin content) on the net charge of the asphaltenes. Electro-deposition experiments were repeated varying one operating parameter while keeping the others constant. The electrode upon which deposition occurred is noted in the final column to the right ${ }^{a}$

\begin{tabular}{|c|c|c|c|c|}
\hline & $\begin{array}{l}\text { Asphaltene and } \\
\text { resin content }\end{array}$ & Oil fraction (vol\%) & Field strength $\left(\mathrm{V} \mathrm{cm}^{-1}\right)$ & Electrode \\
\hline \multirow[t]{3}{*}{ Field strength } & LAS & 15 & 6000 & Cathode \\
\hline & LAS & 15 & 8000 & Cathode \\
\hline & LAS & 15 & 9333 & Both \\
\hline \multirow[t]{3}{*}{ Oil fraction } & LAS & 5 & 8000 & Cathode \\
\hline & LAS & 10 & 8000 & Cathode \\
\hline & LAS & 20 & 8000 & Both \\
\hline \multirow[t]{3}{*}{ Asphaltene and resin content } & HAS & 5 & 6000 & Cathode \\
\hline & HAS & 15 & 8000 & Both \\
\hline & HAS & 20 & 9333 & Both \\
\hline
\end{tabular}

$20 \mathrm{vol} \%$, deposition onto both electrodes was observed. The effect of high $v s$. low asphaltene and resin content can be noted by comparing the third row with the previous two rows. The higher asphaltene and resin content exhibited electro-deposition onto both electrodes indicating a change in the net charge of the aggregate, while only cathode deposition occurred for the LAS. In this study, in no case was deposition onto only the anode observed, which is in contradiction with the Khvostichenko study. ${ }^{21}$ This may be due to the limited electric field strength range (i.e., the voltage supply used) or the different characteristics of the crude oil used to prepare the two blends.

To investigate this further, asphaltenes were separated from the resin and both were purified according to ASTM IP143 procedure and dissolved within a synthetic oil having the same saturate/aromatic ratio (heptane as saturate and toluene as aromatic). The sample was diluted with pentane and the same electro-deposition experiment was conducted. Interestingly, asphaltenes exhibited both a positive and a negative net charge when resin was excluded from the system. Using a low electric field and low oil fraction ( $4000 \mathrm{~V} \mathrm{~cm}^{-1}$ with $20 \mathrm{vol} \%$ oil), deposition onto the cathode was observed, while anode deposition can be triggered when a higher electric field and higher oil fraction $\left(8000 \mathrm{~V} \mathrm{~cm}^{-1}\right.$ with $40 \mathrm{vol} \%$ oil) were used. It is noted that when anode deposition occurred, a relatively high current density was also measured, indicating that the change in the net charge of asphaltenes from positive (cathode deposition) to negative (anode deposition) may related to electron flow. ${ }^{33}$ When resin was introduced into the system, it significantly inhibited the change in the net charge of the asphaltene and only cathode deposition was observed. The adsorption of resin on the asphaltene aggregates may shield the aggregate from the electron flow and require a higher electric field strength to trigger the change in the net charge of the aggregate (anode deposition).

\section{Electric field strength required for complete deposition}

The electric field strength required for complete deposition of various oil volume fractions for both LAS and HAS are plotted in Fig. 5.

For both LAS and HAS, the electric field strength required for complete deposition increased linearly with increasing oil volume fraction, from about $2500 \mathrm{~V} \mathrm{~cm}^{-1}$ for $5 \mathrm{vol} \%$ oil to more than $10000 \mathrm{~V} \mathrm{~cm}^{-1}$ for $25 \mathrm{vol} \%$ oil. A higher viscosity corresponds with higher oil fraction, which may partially explain the higher electric field strength required. For example, the viscosity of diluted oil with $95 \mathrm{vol} \%$ and $70 \mathrm{vol} \%$ were 1.8 and $2.1 \mathrm{cP}$, respectively. In addition, the ratio of the net charge to the mass of the aggregate should also strongly affect the results. With higher oil volume fraction, more resin is associated with the aggregate, likely resulting in a lower charge to mass ratio and thus, a higher electric field strength required for complete deposition.

In this study, the electric field strength required in order to merely observe deposition was much lower than that required for complete deposition. For example, a $2000 \mathrm{~V} \mathrm{~cm}^{-1}$ field strength was required for $20 \mathrm{vol} \%$ oil fraction to observe deposition with LAS, which increased to $5500 \mathrm{~V} \mathrm{~cm}^{-1}$ required for 30 vol\% oil fraction. Also, it was noted that, during the electro-deposition, aggregates accumulated near the electrodes but were not deposited. The "near-electrode" aggregates appeared to decrease the mean free path of the aggregates, thereby increasing the field strength required to pull all aggregates onto the electrodes. Due to the upper limit of the voltage supply, no trials were conducted above $25 \% \mathrm{v} / \mathrm{v}$ oil fraction for HAS and the single data point for $30 \% \mathrm{v} / \mathrm{v}$ for HAS was estimated

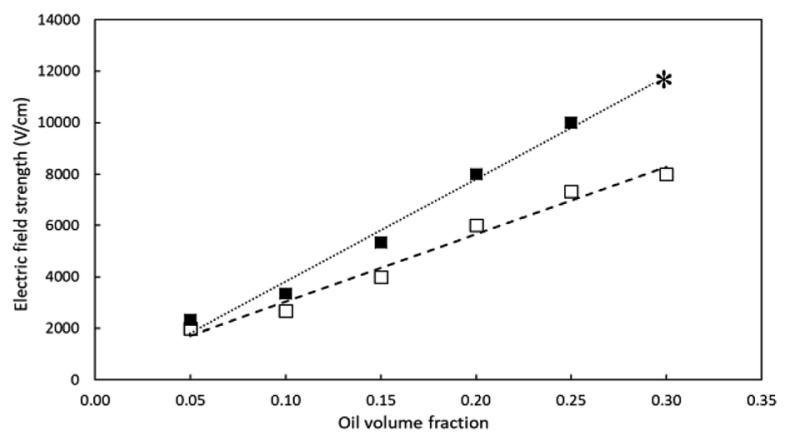

Fig. 5 The electric field strength required for completed the electrodeposition of asphaltenes with various oil volume fraction; white: LAS crude oil; black: HAS crude oil. *Data point was calculated with linear data regression. 
Table 3 Parameters used to estimate energy consumption of electrodeposition process
Electric field strength $(V)$

Permittivity of a dielectric $(\varepsilon)$

Area of electrode $(A)$

Distance between the electrode $(d)$

Process time $(t)$

Mass of asphaltene $\left(m_{\mathrm{a}}\right)$
Average current (I)
$6000 \mathrm{~V}$

$1.59 \times 10^{-11} \mathrm{~F} \mathrm{~m}^{-1}$

$6.67 \times 10^{-4} \mathrm{~m}^{2}$

$0.015 \mathrm{~m}$

$2.70 \times 10^{-7} \mathrm{~A}$

$60 \mathrm{~s}$

$1.47 \times 10^{-5} \mathrm{~kg}$ using linear extrapolation. It is reasonable to extrapolate the data obtained at the lower oil fractions, where the required electric field strength was linear with increasing oil fraction, and assume that the asphaltenes would be deposited from a higher oil volume fraction dilution if a stronger electric field strength were applied.

\section{Energy consumption}

To calculate the energy input of the process, the parameters of the case (LAS blend diluted with 95\% pentane under 6000 $\mathrm{V} \mathrm{cm}^{-1}$ ) of the study were summarized in Table 3 .

During the electro-deposition, $1.27 \times 10^{-5} \mathrm{~J}$ was input to build up the electric field strength, following $9.72 \times 10^{-2} \mathrm{~J}$ was input to maintain the electric kinetics of asphaltene aggregation. After the process, $14.7 \mathrm{mg}$ of asphaltene was recovered, requiring $6.61 \mathrm{~J}$ of energy per gram of asphaltene deposited. According to the calculation, most energy was consumed during the asphaltene electrophoresis, which is inversely proportional to the solvent resistivity. Generally speaking, dissolved asphaltene and resin function as electron conductors, which can lower the resistivity. As a result, less energy would be required for light, dilute oil. Collecting the deposited asphaltene could result in a beneficial use by using it as a fuel in a simple combustion generator to provide the energy needed for the E-D process. Considering the combustion heat of asphaltenes is 40 $\mathrm{kJ} \mathrm{g}^{-1}$, the recovered asphaltenes can generate approximately $230 \mathrm{~J}$ (assuming a conservative 40\% energy conversion efficiency) to meet the energy input demand.

\section{Conclusions}

The precipitation of asphaltene that occurs during oil upgrading can be recovered through the electro-deposition method. As only precipitated asphaltene showed electrophoretic mobility, the assembled structure of asphaltene was necessary for asphaltene to maintain net charge. High diluent ratio results in better electro-deposition performance, meaning a higher fraction of asphaltene precipitated and requiring lower electric field strength to complete the deposition. The net charge of the asphaltenes exhibited during the E-D was determined both the process parameters and the interaction between asphaltene and resin. By affecting the fluid viscosity and conductivity, the asphaltene content in the upgraded oil could significant affect the amount of energy input. The process is highly energy efficiency, and the energy required can be produced by means of recycling the asphaltenes to be used for energy generation. With nominal effect on the flow of the upgraded oil and a high asphaltenes removal efficiency, electro-deposition is a promising approach to asphaltene removal in oil upgrading.

\section{Conflicts of interest}

The authors have no conflicts of interest.

\section{Acknowledgements}

The authors would like to thank Division of Research, University of Houston for the financial support for the work. We would also like to thank Dr Adry Bissada and his group in the Center for Petroleum Geochemistry, Department of Earth and Natural Sciences at the University of Houston for the SARA analysis work and $\mathrm{Mr}$ Gerald Blosser at the University of Houston for the manufacture of Teflon ${ }^{\circledR}$ vessel used for these experiments.

\section{References}

1 S. K. Das, SPE J., 1998, 3, 232-237.

2 A. Shah, R. Fishwick, J. Wood, G. Leeke, S. Rigby and M. Greaves, Energy Environ. Sci., 2010, 3, 700-714.

3 A. B. Bazyleva, M. A. Hasan, M. Fulem, M. Becerra and J. M. Shaw, J. Chem. Eng. Data, 2009, 55, 1389-1397.

4 R. Pal and E. Rhodes, J. Rheol., 1989, 33, 1021-1045.

5 I. M. Krieger and T. J. Dougherty, Trans. Soc. Rheol., 1959, 3, 137-152.

6 P. Luo and Y. Gu, Fuel, 2007, 86, 1069-1078.

7 A. Hart, J. Pet. Explor. Prod. Technol., 2014, 4, 327-336.

8 A. I. Victorov and A. Firoozabadi, AIChE J., 1996, 42, 17531764.

9 J. Long, B. Shen, H. Ling, J. Zhao and J. Lu, Ind. Eng. Chem. Res., 2011, 50, 11259-11269.

10 I. Yudin, G. Nikolaenko, E. Gorodetskii, V. Kosov, V. Melikyan, E. Markhashov, D. Frot and Y. Briolant, J. Pet. Sci. Eng., 1998, 20, 297-301.

11 S. M. Hashmi and A. Firoozabadi, Soft Matter, 2012, 8, 18781883.

12 K. Qian, A. S. Mennito, K. E. Edwards and D. T. Ferrughelli, Rapid Commun. Mass Spectrom., 2008, 22, 2153-2160.

13 B. Schuler, S. Fatayer, G. Meyer, E. Rogel, M. Moir, Y. Zhang, M. R. Harper, A. E. Pomerantz, K. D. Bake and M. J. E. Witt, Energy Fuels, 2017, 31, 6856-6861.

14 B. Schuler, G. Meyer, D. Peña, O. C. Mullins and L. Gross, J. Am. Chem. Soc., 2015, 137, 9870-9876.

15 A. Hosseini, E. Zare, S. Ayatollahi, F. M. Vargas, W. G. Chapman, K. Kostarelos and V. Taghikhani, Fuel, 2016, 178, 234-242.

16 D. S. Khvostichenko and S. I. Andersen, Energy Fuels, 2009, 23, 811-819.

17 F. J. Martín-Martínez, E. H. Fini and M. J. Buehler, RSC Adv., 2015, 5, 753-759.

18 L. Rejon, O. Manero and C. Lira-Galeana, Fuel, 2004, 83, 471476.

19 H. Taghavi, S. Ashoori and S. H. Mousavi, Pet. Sci. Technol., 2018, 36, 487-493. 
20 J. Murgich, J. A. Abanero and O. P. Strausz, Energy Fuels, 1999, 13, 278-286.

21 D. S. Khvostichenko and S. I. Andersen, Energy Fuels, 2010, 24, 2327-2336.

22 H. Asaadian, B. Soltani Soulgani and A. Karimi, Pet. Sci. Technol., 2017, 35, 2255-2261.

23 J. D. McLean and P. K. Kilpatrick, J. Colloid Interface Sci., 1997, 189, 242-253.

24 F. Nellensteyn, The Science of Petroleum, 1938, vol. 4.

25 M. Sedghi and L. Goual, Energy Fuels, 2009, 24, 2275-2280.

26 K. Akbarzadeh, H. Alboudwarej, W. Y. Svrcek and H. W. Yarranton, Fluid Phase Equilib., 2005, 232, 159-170.

27 C. Reichardt and T. Welton, Solvents and solvent effects in organic chemistry, John Wiley \& Sons, 2011.
28 L. C. Palermo and E. F. Lucas, Energy Fuels, 2016, 30, 39413946.

29 M. Mousavi, T. Abdollahi, F. Pahlavan and E. H. Fini, Fuel, 2016, 183, 262-271.

30 V. Azari, E. Abolghasemi, A. Hosseini, S. Ayatollahi and F. Dehghani, Colloids Surf., A, 2018, 541, 68-77.

31 W. Jia, S. Chen, X. Zhu, P. a. Peng and Z. Xiao, J. Anal. Appl. Pyrolysis, 2017, 126, 99-104.

32 A. Boytsova, N. Kondrasheva and J. Ancheyta, Energy Fuels, 2018, 32, 1132-1138.

33 C. Schreuer, S. Vandewiele, F. Strubbe, K. Neyts and F. Beunis, J. Colloid Interface Sci., 2018, 515, 248-254. 